\title{
Development of Semiconductor Detectors for Fast Neutron Radiography
}

\author{
R. T. Klann ${ }^{\mathrm{a}}$, C. L. Fink ${ }^{\mathrm{a}}$, D. S. McGregor ${ }^{\mathrm{b}}$, and H. K. Gersch ${ }^{\mathrm{b}}$ \\ ${ }^{a}$ Technology Development Division, Argonne National Laboratory, Argonne, IL 60439 USA \\ ${ }^{b}$ Department of Nuclear Engineering and Radiological Sciences, University of Michigan, \\ Ann Arbor, MI 48109 USA
}

\begin{abstract}
A high-energy neutron detector has been developed using a semiconductor diode fabricated from bulk gallium arsenide wafers with a polyethylene neutron converter layer. Typical thickness of the diode layer is 250 to $300 \mu \mathrm{m}$ with bias voltages of 30 to 150 volts. Converter thicknesses up to $2030 \mu \mathrm{m}$ have been tested. GaAs neutron detectors offer many advantages over existing detectors including positional information, directional dependence, gamma discrimination, radiation hardness, and spectral tailoring. Polyethylene-coated detectors have been shown to detect $14 \mathrm{MeV}$ neutrons directly from a D-T neutron generator without interference from gamma rays or scattered neutrons. An array of small diode detectors can be assembled to perform fast neutron radiography with direct digital readout and real-time display of the image produced. In addition, because the detectors are insensitive to gamma rays and low energy neutrons, highly radioactive samples (such as spent nuclear fuel or transuranic waste drums) could be radiographed.
\end{abstract}

\section{INTRODUCTION}

Neutron radiography is a well developed and commercially viable technique that is used throughout the world for non-destructive examinations. The technique employs a neutron source and collimators to produce a neutron beam. A sample is then placed in the neutron beam and recording media are placed directly behind the sample. The attenuation of the beam by the sample produces a two-dimensional "shadow image" of the sample. A more detailed description of neutron radiography can be found in the literature $[1,2]$.

Thermal and epithermal neutron radiography are the most widely used forms of neutron radiography. These utilize low-energy neutrons which have a limited range in most materials of interest. Fast neutron radiography, i.e. utilizing neutrons with energies around $14 \mathrm{MeV}$ produced from the D-T reaction, has been explored as a non-destructive examination tool for larger samples because of the greater penetrability of the neutrons [3-7]. The recording media at these energies has been almost exclusively a proton-producing plastic coupled with $\mathrm{X}$-ray scintillation screens. A sheet of light-sensitive film is placed in contact with the scintillation screens to record a latent image on the film. The film is then removed and chemically processed to produce a radiograph. This process has been shown to produce reasonable radiographs, but it has serious limitations. The technique is slow because of the time it takes to produce the image (exposure time) and the time it takes to develop the film. The technique is inconsistent because of the chemical processing. In addition, the processing requires specialized equipment and chemicals which introduce environmental and industrial hazards for use and disposal. The imaging screens and film are sensitive to gamma radiation and $X$ rays which means that the screens and film must be shielded (with some neutron loss) and samples cannot be radioactive. Another drawback is that the images produced are not digital. The radiograph must be scanned to produce a digital image with a loss in resolution and contrast due to limitations in scanning technology.

This paper discusses the development of a new type of coated semiconductor detector for the detection of fast neutrons. Small contacts are deposited on semiinsulating bulk gallium arsenide (GaAs) wafers and then coated with a hydrogen-rich material, e.g. polyethylene. A direct read-out of the detector count rate is obtained with standard electronics. This configuration allows multiple diodes to be created using a single wafer such that positional information 
can be obtained and used to produce an image $[8,9]$. The resolution of the radiography is limited by the size of the individual diodes.

\section{THEORY OF OPERATION}

When a voltage is applied across the GaAs wafer, a truncated high field or active region is produced near the contact [10]. The incident neutrons are converted into charged particles in the coating, i.e. recoil protons, which excite free charge carriers in the GaAs detector active region. The charge carriers are drifted to the detector contacts, and a preamplifier circuit measures the induced charge. Charges excited in the low field or substrate region are not collected. In addition, because the active region is so thin $(10-20 \mu \mathrm{m})$, background gamma-ray interactions are reduced. Gamma rays that are absorbed in the active region are easily discriminated from the recoil protons. Figure 1 is a schematic representation.

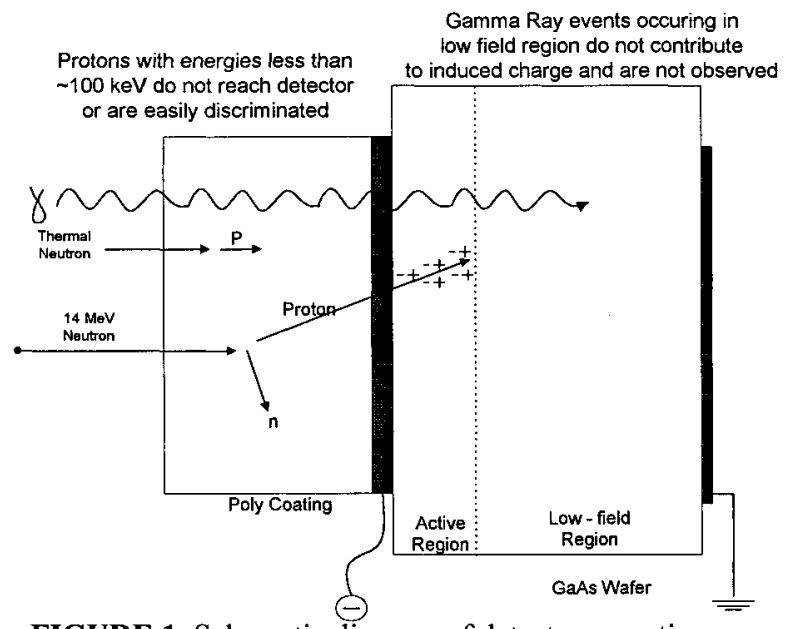

FIGURE 1. Schematic diagram of detector operation

\section{DETECTOR FABRICATION}

Schottky barrier bulk GaAs diodes were fabricated with high-density polyethylene (HDP) coatings. The GaAs diodes were manufactured in the following manner. Commercial bulk semi-insulating (SI) GaAs wafers were used for the devices. The back surfaces were lapped at $30 \mathrm{rpm}$ with a $3 \mu \mathrm{m}$ calcined aluminum oxide powder/ deionized water solution over an optically flat glass plate until $100 \mu \mathrm{m}$ of GaAs material was removed. Afterwards, the backsides were polished with $0.3 \mu \mathrm{m}$ calcined aluminum oxide powder mixed in a sodium hypochlorite solution over a chemically resistant polishing cloth at $65 \mathrm{rpm}$ for 10 minutes. A final polish was performed with a 50:50:1 methyl alcohol:glycerol:bromine solution for 10 minutes over a chemically resistant polishing pad at $70 \mathrm{rpm}$. The wafers were cleaned in a series of solvents and etched in a $1: 1: 320 \mathrm{H}_{2} \mathrm{SO}_{4}: \mathrm{H}_{2} \mathrm{O}_{2}$ :deionized water solution for 5 minutes followed by a 2 minute etch in a $1: 1 \mathrm{HCl}$ : deionized water solution. The wafers were then cleaned in a deionized water cascade and blown dry with $\mathrm{N}_{2}$.

The backsides were implanted at an angle of $7^{\circ}$ from normal with ${ }^{29} \mathrm{Si}$ ions at an average energy of 100 $\mathrm{keV}$ at a dose amounting to $5 \times 10^{13}$ ions per $\mathrm{cm}^{2}$. The implants were activated with a rapid thermal anneal in Ar for 30 seconds at $800^{\circ} \mathrm{C}$. Afterwards, a stacked layer of $\mathrm{Ge}(500 \AA)$ : $\mathrm{Pd}(1300 \AA)$ was vacuum evaporated over the backsides, followed by a low temperature anneal of $250^{\circ} \mathrm{C}$ in $\mathrm{N}_{2}$ for 30 minutes. Vacuum evaporation of a stacked layer of Ti (150 $\AA$ ): Au (700 $\AA$ ) completed the backside processing of the devices.

Front-side processing of the devices included lapping and polishing of the samples, in which the initial lapping with $3 \mu \mathrm{m}$ calcined aluminum oxide powder was used to thin the wafers to $250 \mu \mathrm{m}$ total thickness. Afterwards, the wafers were polished using the $0.3 \mu \mathrm{m}$ calcined aluminum oxide powder mixed in a sodium hypochlorite solution followed by the methyl alcohol:glycerol:bromine solution. Again, the wafers were cleaned in a series of solvents and etched in a 1:1:320 $\mathrm{H}_{2} \mathrm{SO}_{4}: \mathrm{H}_{2} \mathrm{O}_{2}$ :deionized water solution for 5 minutes followed by a 2 minutes etch in a 1:1 $\mathrm{HCl}$ :deionized water solution. Afterwards, the wafers were cleaned in a deionized water cascade and blown dry with $\mathrm{N}_{2}$.

The basic pad area designs were patterned onto the surfaces with photoresist. A final etch in the patterns was performed with the $\mathrm{H}_{2} \mathrm{SO}_{4}: \mathrm{H}_{2} \mathrm{O}_{2}$ :deionized water solution followed by the $\mathrm{HCl}$ :deionized water solution. The wafers were washed in a deionized water cascade and blown dry with $\mathrm{N}_{2}$. A stacked layer of Ti:Au was evaporated over the wafer and lifted off in acetone. Other variations used a system of Ti:Pt:Au contacts.

Polyethylene-coated devices were manufactured by adhering various thicknesses of HDP sheets to the bare Schottky contacts. The HDP sample thicknesses included "Humiseal" only, $50 \mu \mathrm{m}$ (0.002 inches), 75 $\mu \mathrm{m}$ (0.003 inches), $125 \mu \mathrm{m}$ (0.005 inches), $250 \mu \mathrm{m}$ (0.010 inches), $450 \mu \mathrm{m}$ (0.018 inches), $900 \mu \mathrm{m}(0.035$ inches), and $2030 \mu \mathrm{m}$ (0.080 inches). The individual 
devices were cleaved from the GaAs wafers, and fastened with silver-based epoxy to $1 \mathrm{~mm}$ thick aluminum oxide mounts. Figure 2 shows several detectors with different coatings. The detector area, defined by the diode size, is a $25 \mathrm{~mm}^{2}$ circle with one diode on each detector.

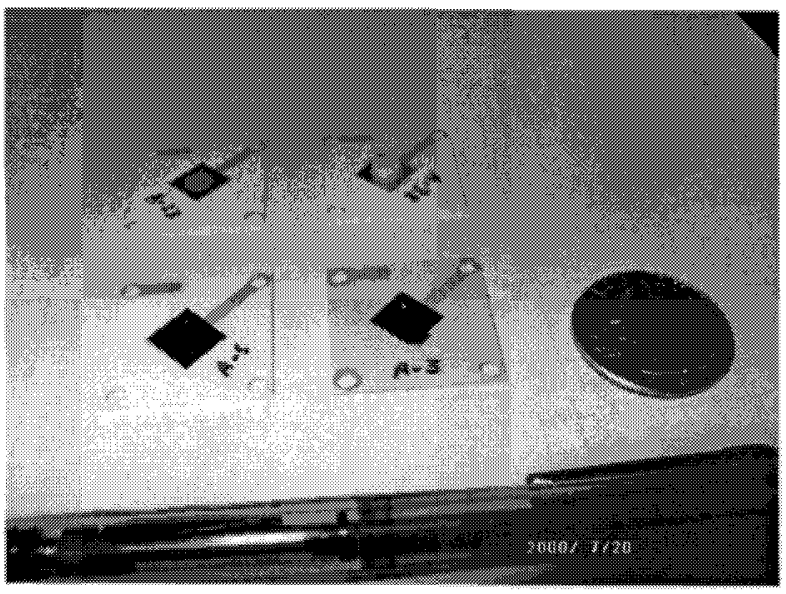

FIGURE 2: GaAs Detectors on Alumina mounts

\section{TEST RESULTS}

The GaAs Schottky barrier detectors were mounted in light-impenetrable $\mathrm{Al}$ boxes. The enclosed devices were then placed at a distance of $10 \mathrm{~cm}$ from the target cooling cap of an MF Physics A-711 Neutron Generator. The detectors were operated with reverse bias voltages between 30 and 150 volts.

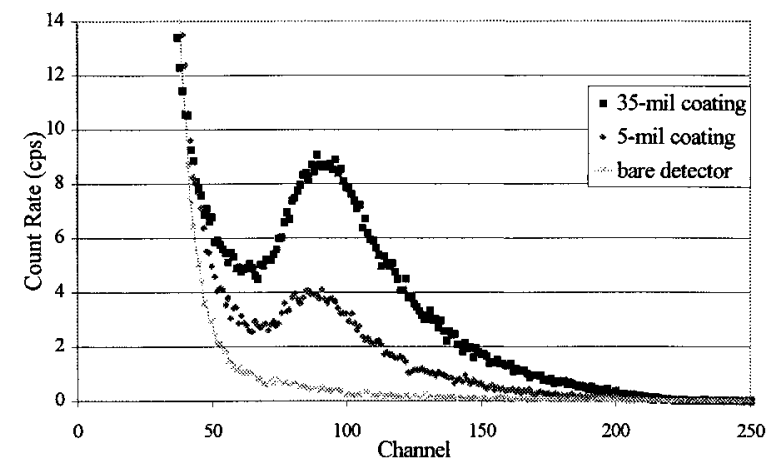

FIGURE 3: Fast neutron response for two different coating thicknesses (detector bias of 120 volts).

Figure 3 shows the results for coating thicknesses of 0.005 ( 5 mils) and 0.035 inches ( 35 mils) compared to the results from an uncoated detector for a bias of 120 volts. The peak around channel 90 is a result of the recoil proton energy deposited in the active region of the detector. The low energy tail is due to detector noise and background from gamma rays, $X$ rays, and scattered neutrons. In practice, the lower level discriminator would be set in the valley of the spectrum to maximize the neutron signal to noise ratio. The relative efficiency of the 35 -mil coating is 2.7 times that of the 5-mil coating. This is less than predicted from modeling studies [11] and is likely the result of energy straggling, edge effects, and effects from the contact materials, which were not accounted for in the modeling studies.

The range of the recoil protons exceeds the high field region of the detector. By increasing the bias on the detector, the high field region is increased, leading to higher energy deposition and a larger pulse from the detector. This can be observed in Figure 4 and Table 1. Figure 4 shows the results of increasing the detector bias while maintaining all other parameters constant. The entire curve is amplified due to the increase in the depth of the active region of the detector. The effect becomes apparent in Table 1 . The neutron count rate is the total count rate of all channels above the valley. It is relatively constant ( $12 \%$ change) over the entire range of bias voltages while the total count rate has increased by a factor of 15 . The noise is being amplified by the higher voltage and the gamma response is being increased because of the increase in active region.

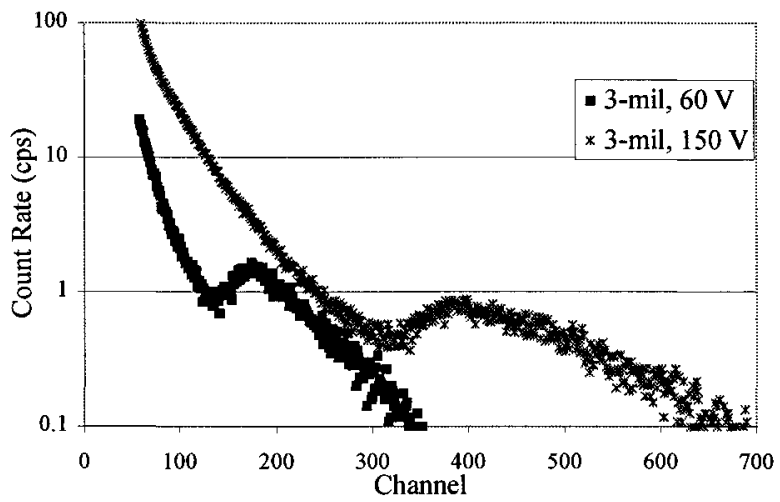

FIGURE 4: Fast neutron response for two different detector bias voltages.

\begin{tabular}{|c|c|c|c|c|}
\hline \multicolumn{5}{|c|}{ Table 1 } \\
\hline $\begin{array}{c}\text { Bias } \\
\text { Voltage } \\
\text { (volts) }\end{array}$ & $\begin{array}{c}\text { Total } \\
\text { Rate } \\
\text { (cps) }\end{array}$ & $\begin{array}{c}\text { Neutron } \\
\text { Rate } \\
\text { (cps) }\end{array}$ & $\begin{array}{c}\text { Valley } \\
\text { Channel }\end{array}$ & $\begin{array}{c}\text { Peak } \\
\text { Channel }\end{array}$ \\
\hline 30 & 211.6 & 138.7 & 85 & 100 \\
\hline 60 & 502.7 & 141.0 & 140 & 175 \\
\hline 90 & 1211.4 & 147.2 & 195 & 255 \\
\hline 120 & 2103.8 & 154.2 & 250 & 330 \\
\hline 150 & 3182.0 & 157.8 & 320 & 380 \\
\hline
\end{tabular}




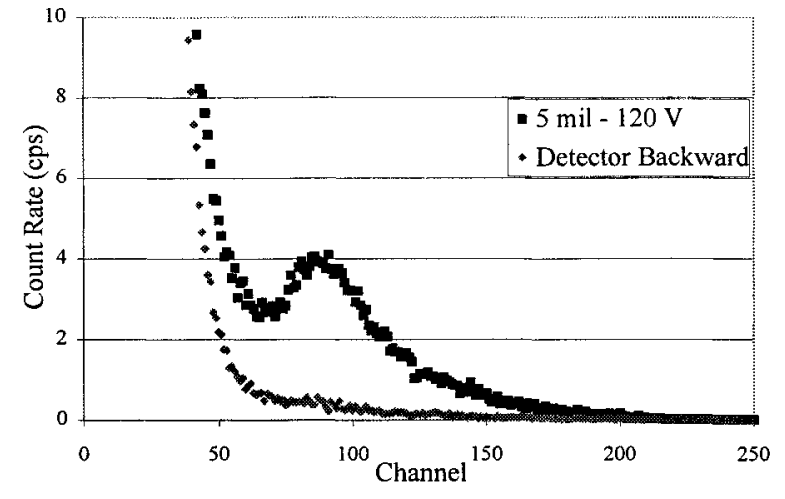

FIGURE 5: Directional response of 5-mil coated GaAs detectors to fast neutrons

Figure 5 compares the response of a detector facing the neutron source to that of a detector facing away from the neutron source. Figure 5 establishes that the device is directionally sensitive, in which the forward scattered recoil protons can enter the GaAs detector only if it is facing the general direction of the fast neutron source. If the response were due to any other reaction or radiation incident on the detector, the spectrum for the backward facing detector would show a response. Instead, the response of the backward facing detector is statistically identical to the uncoated detector response shown in Figure 3. This also demonstrates that there is a negligible response from lower energy neutrons due to scattering in the room. For radiography purposes, this helps to reduce background and eliminates the need for collimation or shielding of the detectors. In addition, it helps to improve image resolution and clarity because scattered neutrons simply do not contribute to the neutron signal.

\section{CONCLUSIONS}

Semiconductor diode detectors using bulk GaAs wafers have been fabricated and tested. The detectors have been shown to respond to fast neutrons with minimal response from scattered neutrons and gamma rays. With energy discrimination these effects can be easily reduced such that a clean signal is obtained for the fast neutron response. In addition, the detectors have been shown to be directionally dependent such that the response from background neutrons is significantly reduced.

The detectors are digital, in that the total count rate is summed to produce a numeric value for a given detector. With an array of these types of diodes, a digital fast neutron radiograph could be produced. The resolution of such a system would be dependent on the size of the individual diodes. Diodes have been produced with areas much smaller than $1 \mathrm{~mm}^{2}$, which means that this approach is viable for fast neutron radiography.

\section{ACKNOWLEDGMENTS}

The submitted manuscript has been authored by a contractor of the U.S. Government under contract No. W-31-109-ENG-38. Accordingly, the U.S. Government retains a nonexclusive, royalty-free license to publish or reproduce the published form of this contribution, or allow others to do so, for U.S. Government purposes. Research at the University of Michigan was performed under an appointment to the U.S. Department of Energy Nuclear Engineering and Health Physics Fellowship Program sponsored by DOE Office of Nuclear Energy, Science, and Technology.

\section{REFERENCES}

1. Von Der Hardt, P., Rottger, H., Neutron Radiography Handbook, D. Reidel Publishing Company, London, 1981.

2. Berger, H., Iddings, F., Neutron Radiography: A Stateof-the-Art Report, Non-Destructive Testing Information Analysis Center Report No. NTIAC-SR-98-01, TRI/Austin, Inc., 1998.

3. Klann, R. and Natale, M., "Fast Neutron Radiography Research at ANL-W," Proceedings from the Fifth World Conference on Neutron Radiography, edited by C.O. Fischer, et al., DGZfP BB53, Berlin, Germany, 1997, pp.382-390.

4. Klann, R., "Fast Neutron (14.5 MeV) Radiography: A Comparative Study," Proceedings from the Fifth World Conference on Neutron Radiography, edited by C.O. Fischer, et al., DGZfP BB53, Berlin, Germany, 1997, pp.469-483.

5. Richardson, A., Materials Evaluation 35, 52-58 (1977).

6. Brzosko, J., et al., Nucl. Inst. Meth. Phys. Res. B72, 119131 (1992).

7. Kim, K., et al., Nucl. Inst. Meth. Phys. Res. A422, 929932 (1999).

8. McGregor, D., et al., IEEE Trans. Nucl. Sci. 43, 13571364 (1996).

9. Manolopoulos, S., et al., IEEE Trans. Nucl. Sci. 45, 394-400 (1998).

10. McGregor, D., and Kammeraad, J., Semiconductors and Semimetals, 43, 383-442, (1995).

11. Klann, R., and McGregor. D., "Development of Coated Gallium Arsenide Neutron Detectors," Proceedings from the Eighth International Conference on Nuclear Engineering (ICONE-8), edited by S. Anghaie, et al., ASTM, New York, New York, ICONE-81 10, pp. 1-6. 